\title{
Collocation Methods for Pricing American Strangle Options
}

\author{
Jingtang Ma \& Youjin Zhang \\ School of Economic Mathematics, Southwestern University of Finance and Economics \\ Chengdu 611130, China \\ E-mail: mjt@swufe.edu.cn
}

Received: March 16, 2012

Accepted: April 3, 2012

Published: May 15, 2012

doi:10.5539/afr.v1n1p207

URL: http://dx.doi.org/10.5539/afr.v1n1p207

The work was supported by the Fundamental Research Funds for the Central Universities.

\begin{abstract}
The aim of this paper is to develop high-order collocation methods for pricing American strangle options. The major difficulty in pricing American strangles is to determine the optimal exercise boundaries. Chiarella and Ziogas (2005) derived that the optimal exercise boundaries satisfy a system of integral equations. Since the analytical solutions of the integral equation system cannot be found, it relies on numerical methods to solve the integral equation system. In the literature, there are no efficient and reliable numerical methods for solving the integral equation system. This paper develops a high-order collocation method to solve the integral equation system. Numerical example is carried out to show that the collocation methods are much more reliable and efficient.
\end{abstract}

Keywords: American strangle options, Optimal exercise boundaries, Collocation methods, Integral equations

\section{Introduction}

Pricing options has a long history (see a survey paper: Broadie and Detemple (2004)). As well-known, the key to pricing American options is to identify the optimal exercise boundary. Since the close-form of optimal exercise boundary cannot be obtained in general, numerical methods become the necessary tools to resolve the problems. One of the numerical methods for pricing American options is the integral equation approach. The basic idea of this approach is to derive an integral equation or integral equation system for the optimal exercise boundaries and then to solve the integral equations to get the approximate solutions. The integral equations can be derived by the EEP representations: American option price is equal to the corresponding European option price plus an early exercise premium (EEP). In the history, Kim (1990), Jacka (1991), and Carr et al. (1992) derived the EEP representations for standard American options with the underlying asset price following a lognormal process, and Detemple and Tian (2002) provided the EEP representation under general diffusion process with stochastic volatility and interest rate. Huang et al. (1996), Ju (1998), Detemple and Tian (2002) studied the implementations of the EEP methods for pricing the standard American options. However their approaches for solving the integral equations are based on low-order approximations and not fast. Recently Ma et al. (2010) constructed a high-order collocation method on non-uniform meshes for solving the integral equation arising in the EEP representation of American put options under lognormal process. Ma et al. (2011) extended the approach to pricing standard American options with stochastic volatility.

In this paper, we consider the valuation of American strangle options which consist of two sides, namely call side and put side, automatically knocking out the other side when exercising one side. Chiarella and Ziogas (2005) derived a system of integral equations for the optimal exercise boundaries. We aim at developing collocation methods (see Brunner (2004)) to solve the system of integral equations. Numerical examples show that the collocation methods are three times faster than Chiarella and Ziogas's implementations. In the history, in addition to Chiarella and Ziogas (2005), there are other papers discussing American straddles (a special case of American strangles with one strike price) : Elliott, Myneni and Viswanathan (1990) derived an integral equation system for the optimal exercise boundaries for American straddle; Alobaidi and Mallier (2002) used Laplace transform to price American straddle; Boyarchenko (2006) studied perpetual American straddle under jump-diffusion process. Detemple and Emmerling (2009) studied a similar type of American options --- American chooser options.

The remaining parts are arranged as follows: In the next section we describe the mathematical problems; In Section 3, we introduce the collocation methods; In Section 4, we provide numerical examples to illustrate the efficiency of our 
approach. In the final section, we give a concluding remark.

\section{Mathematical problems for pricing American strangles}

Assume that the price of the underlying assets follows a geometric Brownian process

$$
\mathrm{dS}=(\mathrm{r}-\mathrm{q}) \mathrm{Sdt}+\sigma \mathrm{SdW}_{\mathrm{t}}
$$

where $\mathrm{r}$ is riskless interest rate, $\mathrm{q}$ dividend rate, $\sigma$ volatility, and $\mathrm{W}_{\mathrm{t}}$ Brownian motion. American strangle option has two strike prices: $\mathrm{K}_{1}$ (put-side strike) and $\mathrm{K}_{2}$ (call-side strike), $\mathrm{K}_{1}<\mathrm{K}_{2}$ and the payoff

$$
\mathrm{V}(\mathrm{S}, \mathrm{T})=\max \left(\mathrm{K}_{1}-\mathrm{S}, 0\right)+\max \left(\mathrm{S}-\mathrm{K}_{2}, 0\right) .
$$

When $\mathrm{K}_{1}=\mathrm{K}_{2}$, the American strangle is called American straddle. As shown in Figure 1, the exercise region for American strangle has two optimal exercise boundaries, $a_{1}(t)$ and $a_{2}(t)$. Using the framework of Black and Scholes (1973), we can derive that the value of American strangle satisfies the following partial differential equations (PDEs)

$$
\mathrm{V}_{\mathrm{t}}+\left(\sigma^{2} \mathrm{~S}^{2} / 2\right) \mathrm{V}_{\mathrm{sS}}+(\mathrm{r}-\mathrm{q}) \mathrm{SV}_{\mathrm{s}}-\mathrm{rV}=0
$$

for $\mathrm{a}_{1}(\mathrm{t})<\mathrm{S}<\mathrm{a}_{2}(\mathrm{t})$, with the following conditions

(1) $\mathrm{V}(\mathrm{S}, \mathrm{T})=\max \left(\mathrm{K}_{1}-\mathrm{S}, 0\right)+\max \left(\mathrm{S}-\mathrm{K}_{2}, 0\right), 0<\mathrm{S}<+\infty$.

(2) $\mathrm{V}\left(\mathrm{a}_{1}(\mathrm{t}), \mathrm{t}\right)=\mathrm{K}_{1}-\mathrm{a}_{1}(\mathrm{t}), \mathrm{V}\left(\mathrm{a}_{2}(\mathrm{t}), \mathrm{t}\right)=\mathrm{a}_{2}(\mathrm{t})-\mathrm{K}_{2}, \mathrm{t} \geq 0$.

(3) $\lim _{\mathrm{s} \rightarrow \mathrm{a}_{1}} \mathrm{~V}_{\mathrm{s}}=-1, \quad \lim _{\mathrm{s} \rightarrow \mathrm{a}_{2}} \mathrm{~V}_{\mathrm{s}}=1, \mathrm{t} \geq 0$

Chiarella and Ziogas (2005) used Laplace transform for equation (2) to derive that

$$
\mathrm{V}=\mathrm{V}\left(\mathrm{S}_{\mathrm{t}} ; \mathrm{a}_{1}(\mathrm{t}), \mathrm{a}_{2}(\mathrm{t})\right)=\left[\mathrm{p}_{\mathrm{t}}\left(\mathrm{S}_{\mathrm{t}}\right)+\int_{\mathrm{t}}^{\mathrm{T}} \Phi_{\mathrm{t}}^{1}\left(\mathrm{a}_{1}(\mathrm{~s}), \mathrm{S}_{\mathrm{t}}, \mathrm{s}\right) \mathrm{ds}\right]+\left[\mathrm{c}_{\mathrm{t}}\left(\mathrm{S}_{\mathrm{t}}\right)+\int_{\mathrm{t}}^{\mathrm{T}} \Phi_{\mathrm{t}}^{2}\left(\mathrm{a}_{2}(\mathrm{~s}), \mathrm{S}_{\mathrm{t}}, \mathrm{s}\right) \mathrm{ds}\right],
$$

where $\mathrm{V}$ is the price of American strangle options, $\mathrm{p}_{\mathrm{t}}$ the value of European put options with strike price $\mathrm{K}_{1}$, $\mathrm{c}_{\mathrm{t}}$ the value of European call options with strike price $\mathrm{K}_{2}$, and $\Phi_{\mathrm{t}}^{1}, \Phi_{\mathrm{t}}^{2}$ are the cash flows. The explicit formulas are given below:

$$
\begin{gathered}
\mathrm{p}_{\mathrm{t}}\left(\mathrm{S}_{\mathrm{t}}\right)=\mathrm{K}_{1} \mathrm{e}^{-\mathrm{r}(\mathrm{T}-\mathrm{t})} \mathrm{N}\left(-\mathrm{d}_{2}\left(\mathrm{~S}_{\mathrm{t}}, \mathrm{T}-\mathrm{t} ; \mathrm{K}_{1}\right)\right)-\mathrm{S}_{\mathrm{t}} \mathrm{e}^{-\mathrm{q}(\mathrm{T}-\mathrm{t})} \mathrm{N}\left(-\mathrm{d}_{1}\left(\mathrm{~S}_{\mathrm{t}}, \mathrm{T}-\mathrm{t} ; \mathrm{K}_{1}\right)\right), \\
\mathrm{c}_{\mathrm{t}}\left(\mathrm{S}_{\mathrm{t}}\right)=\mathrm{S}_{\mathrm{t}} \mathrm{e}^{-\mathrm{q}(\mathrm{T}-\mathrm{t})} \mathrm{N}\left(\mathrm{d}_{1}\left(\mathrm{~S}_{\mathrm{t}}, \mathrm{T}-\mathrm{t} ; \mathrm{K}_{2}\right)\right)-\mathrm{K}_{2} \mathrm{e}^{-\mathrm{r}(\mathrm{T}-\mathrm{t})} \mathrm{N}\left(\mathrm{d}_{2}\left(\mathrm{~S}_{\mathrm{t}}, \mathrm{T}-\mathrm{t} ; \mathrm{K}_{2}\right)\right), \\
\Phi_{\mathrm{t}}^{1}\left(\mathrm{a}_{1}(\mathrm{~s}), \mathrm{S}_{\mathrm{t}}, \mathrm{s}\right)=\mathrm{rK} \mathrm{e}_{1} \mathrm{e}^{-\mathrm{r}(\mathrm{s}-\mathrm{t})} \mathrm{N}\left(-\mathrm{d}_{2}\left(\mathrm{~S}_{\mathrm{t}}, \mathrm{s}-\mathrm{t} ; \mathrm{a}_{1}(\mathrm{~s})\right)\right)-\mathrm{qS}_{\mathrm{t}} \mathrm{e}^{-\mathrm{q}(\mathrm{s}-\mathrm{t})} \mathrm{N}\left(-\mathrm{d}_{1}\left(\mathrm{~S}_{\mathrm{t}}, \mathrm{s}-\mathrm{t} ; \mathrm{a}_{1}(\mathrm{~s})\right)\right), \\
\Phi_{\mathrm{t}}^{2}\left(\mathrm{a}_{2}(\mathrm{~s}), \mathrm{S}_{\mathrm{t}}, \mathrm{s}\right)=\mathrm{qS}_{\mathrm{t}} \mathrm{e}^{-\mathrm{q}(\mathrm{s}-\mathrm{t})} \mathrm{N}\left(\mathrm{d}_{1}\left(\mathrm{~S}_{\mathrm{t}}, \mathrm{s}-\mathrm{t} ; \mathrm{a}_{2}(\mathrm{~s})\right)\right)-\mathrm{rK}_{2} \mathrm{e}^{-\mathrm{r}(\mathrm{s}-\mathrm{t})} \mathrm{N}\left(\mathrm{d}_{2}\left(\mathrm{~S}_{\mathrm{t}}, \mathrm{s}-\mathrm{t} ; \mathrm{a}_{2}(\mathrm{~s})\right)\right),
\end{gathered}
$$

where

$$
\begin{aligned}
& \mathrm{d}_{1}\left(\mathrm{~S}_{\mathrm{t}}, \tau ; \gamma\right)=\frac{\ln \left(\mathrm{S}_{\mathrm{t}} / \gamma\right)+\left(\mathrm{r}-\mathrm{q}+\sigma^{2} / 2\right) \tau}{\sigma \sqrt{\tau}}, \\
& \mathrm{d}_{2}\left(\mathrm{~S}_{\mathrm{t}}, \tau ; \gamma\right)=\mathrm{d}_{1}\left(\mathrm{~S}_{\mathrm{t}}, \tau ; \gamma\right)-\sigma \sqrt{\tau} .
\end{aligned}
$$

Replacing $\mathrm{S}_{\mathrm{t}}$ in equation (3) by $\mathrm{a}_{1}(\mathrm{t}), \mathrm{a}_{2}(\mathrm{t})$, we derive the integral equation system for the optimal exercise boundaries:

$$
\begin{aligned}
& \mathrm{K}_{1}-\mathrm{a}_{1}(\mathrm{t})=\mathrm{V}\left(\mathrm{a}_{1}(\mathrm{t}) ; \mathrm{a}_{1}(\mathrm{t}), \mathrm{a}_{2}(\mathrm{t})\right), \\
& \mathrm{a}_{2}(\mathrm{t})-\mathrm{K}_{2}=\mathrm{V}\left(\mathrm{a}_{2}(\mathrm{t}) ; \mathrm{a}_{1}(\mathrm{t}), \mathrm{a}_{2}(\mathrm{t})\right) .
\end{aligned}
$$

In addition the following conditions are associated to the integral equations (4), (5), see Chiarella and Ziogas (2005),

$$
\begin{aligned}
& \mathrm{a}_{1}(\mathrm{~T})=\min \left(\frac{\mathrm{rK}_{1}}{\mathrm{q}}, \mathrm{K}_{1}\right), \\
& \mathrm{a}_{2}(\mathrm{~T})=\max \left(\frac{\mathrm{rK}_{2}}{\mathrm{q}}, \mathrm{K}_{2}\right) .
\end{aligned}
$$

In the following section, we will develop collocation methods to solve the integral equations (4), (5). 


\section{Collocation methods}

Using the following transformation we simplify the integral equations (4), (5) and then the collocation methods are proposed to solve the simplified forms of integral equations. To this end, let $\tau=\mathrm{T}-\mathrm{t}$,

$$
\mathrm{a}_{1}(\mathrm{t})=\mathrm{a}_{1}(\mathrm{~T}-\tau)=\tilde{a}_{1}(\tau), \mathrm{a}_{2}(\mathrm{t})=\mathrm{a}_{2}(\mathrm{~T}-\tau)=\tilde{\mathrm{a}}_{2}(\tau),
$$

and

$$
\mathrm{y}_{1}(\tau)=\ln \tilde{a}_{1}(\tau), \mathrm{y}_{2}(\tau)=\ln \tilde{a}_{2}(\tau)
$$

Then integral equations (4), (5) can be written as

$$
\begin{aligned}
& \mathrm{K}_{1}-\mathrm{e}^{\mathrm{y}_{1}(\tau)}=\tilde{\mathrm{V}}\left(\mathrm{e}^{\mathrm{y}_{1}(\tau)} ; \mathrm{e}^{\mathrm{y}_{1}(\tau)}, \mathrm{e}^{\mathrm{y}_{2}(\tau)}\right), \\
& \mathrm{e}^{\mathrm{y}_{2}(\tau)}-\mathrm{K}_{2}=\tilde{\mathrm{V}}\left(\mathrm{e}^{\mathrm{y}_{2}(\tau)} ; \mathrm{e}^{\mathrm{y}_{1}(\tau)}, \mathrm{e}^{\mathrm{y}_{2}(\tau)}\right),
\end{aligned}
$$

where $\tilde{\mathrm{V}}$ is defined by

$\tilde{\mathrm{V}}\left(\delta_{1}(\tau) ; \delta_{2}(\tau), \delta_{3}(\tau)\right)=\left[\tilde{\mathrm{p}}_{\tau}\left(\delta_{1}(\tau)\right)+\int_{0}^{\tau} \tilde{\Phi}_{\tau}^{1}\left(\delta_{2}(v), \delta_{1}(\tau), v\right) \mathrm{d} v\right]+\left[\tilde{\mathrm{c}}_{\tau}\left(\delta_{1}(\tau)\right)+\int_{0}^{\tau} \tilde{\Phi}_{\tau}^{2}\left(\delta_{3}(v), \delta_{1}(\tau), v\right) \mathrm{d} v\right]$ where

$\tilde{\mathrm{p}}_{\tau}\left(\delta_{1}(\tau)\right)=\mathrm{K}_{1} \mathrm{e}^{-\tau \tau} \mathrm{N}\left(-\mathrm{d}_{2}\left(\delta_{1}(\tau), \tau ; \mathrm{K}_{1}\right)\right)-\delta_{1}(\tau) \mathrm{e}^{-q \tau} \mathrm{N}\left(-\mathrm{d}_{1}\left(\delta_{1}(\tau), \tau ; \mathrm{K}_{1}\right)\right)$,

$\tilde{\mathrm{c}}_{\tau}\left(\delta_{1}(\tau)\right)=\delta_{1}(\tau) \mathrm{e}^{-q \tau} \mathrm{N}\left(\mathrm{d}_{1}\left(\delta_{1}(\tau), \tau ; \mathrm{K}_{2}\right)\right)-\mathrm{K}_{2} \mathrm{e}^{-\mathrm{rt}} \mathrm{N}\left(\mathrm{d}_{2}\left(\delta_{1}(\tau), \tau ; \mathrm{K}_{2}\right)\right)$,

$\left(\delta_{2}(v), \delta_{1}(\tau), v\right)=\mathrm{rK}_{1} \mathrm{e}^{-\mathrm{r}(\tau-v)} \mathrm{N}\left(-\mathrm{d}_{2}\left(\delta_{1}(\tau), \tau-v ; \delta_{2}(v)\right)\right)-\mathrm{q} \delta_{1}(\tau) \mathrm{e}^{-\mathrm{q}(\tau-v)} \mathrm{N}\left(-\mathrm{d}_{1}\left(\delta_{1}(\tau), \tau-v ; \delta_{2}(v)\right)\right)$,

$\tilde{\Phi}_{\tau}^{2}\left(\delta_{3}(v), \delta_{1}(\tau), v\right)=\mathrm{q} \delta_{1}(\tau) \mathrm{e}^{-\mathrm{q}(\tau-v)} \mathrm{N}\left(\mathrm{d}_{1}\left(\delta_{1}(\tau), \tau-v ; \delta_{3}(v)\right)\right)-\mathrm{rK}_{2} \mathrm{e}^{-\mathrm{r}(\tau-v)} \mathrm{N}\left(\mathrm{d}_{2}\left(\delta_{1}(\tau), \tau-v ; \delta_{3}(v)\right)\right)$.

Now we introduce collocation methods to solve integral equations (6) and (7) to get the optimal exercise boundaries for the American strangle options. We first define graded meshes:

$$
\mathrm{t}_{\mathrm{i}}=\mathrm{T}\left(\frac{\mathrm{i}}{\mathrm{L}}\right)^{2}, \quad \mathrm{i}=0,1, \ldots, \mathrm{L}
$$

and piecewise continuous polynomial spaces:

$$
\prod_{3}^{-1}(0, T]=\left\{p: p(t) \in P_{3}, t \in\left(t_{i}, t_{i+1}\right], i=0,1, \ldots, L-1\right\},
$$

where $\mathrm{P}_{3}$ denotes polynomials of degree less than three. The collocation methods are defined as:

Find $Y^{1}(\tau), Y^{2}(\tau) \in \prod_{3}^{-1}$ such that the following equations

hold true at the collocation points

$$
\begin{aligned}
& \mathrm{K}_{1}-\mathrm{e}^{\mathrm{Y}^{1}(\tau)}=\tilde{\mathrm{V}}\left(\mathrm{e}^{\mathrm{Y}^{1}(\tau)} ; \mathrm{e}^{\mathrm{Y}^{\prime}(\tau)}, \mathrm{e}^{\mathrm{Y}^{2}(\tau)}\right), \\
& \mathrm{e}^{\mathrm{Y}^{2}(\tau)}-\mathrm{K}_{2}=\tilde{\mathrm{V}}\left(\mathrm{e}^{\mathrm{Y}^{2}(\tau)} ; \mathrm{e}^{\mathrm{Y}^{1}(\tau)}, \mathrm{e}^{\mathrm{Y}^{2}(\tau)}\right),
\end{aligned}
$$

$$
\mathrm{t}_{\mathrm{i}, \mathrm{j}}=\mathrm{t}_{\mathrm{i}}+\frac{\mathrm{j}}{4}\left(\mathrm{t}_{\mathrm{i}+1}-\mathrm{t}_{\mathrm{i}}\right), \quad \mathrm{j}=1,2,3,4 ; \quad \mathrm{i}=0,1, \ldots, \mathrm{L}-1 .
$$

On the interval $\left(\mathrm{t}_{\mathrm{i}}, \mathrm{t}_{\mathrm{i}+1}\right]$, polynomials $\mathrm{Y}^{1}(\tau), \mathrm{Y}^{2}(\tau)$ can be represented by

$$
\mathrm{Y}^{1}(\tau)=\sum_{\mathrm{j}=1}^{4} \mathrm{Y}_{\mathrm{i}, \mathrm{j}, \mathrm{i}, \mathrm{j}}^{1}(\tau), \quad \mathrm{Y}^{2}(\tau)=\sum_{\mathrm{j}=1}^{4} \mathrm{Y}_{\mathrm{i}, \mathrm{j}}^{2} 1_{\mathrm{i}, \mathrm{j}}(\tau),
$$

where $1_{i, j}(t), j=1,2,3,4$; are Lagrange basis functions,

Inserting (10) into (8) and (9) gives that

$$
1_{i, j}(t)=\prod_{k \neq j} \frac{t-t_{i, k}}{t_{i, j}-t_{i, k}} .
$$

$$
\begin{aligned}
& F_{i, j}^{1}\left(Y_{i, 1}^{1}, Y_{i, 2}^{1}, Y_{i, 3}^{1}, Y_{i, 4}^{1}, Y_{i, 1}^{2}, Y_{i, 2}^{2}, Y_{i, 3}^{2}, Y_{i, 4}^{2}\right)=0, \\
& F_{i, j}^{2}\left(Y_{i, 1}^{1}, Y_{i, 2}^{1}, Y_{i, 3}^{1}, Y_{i, 4}^{1}, Y_{i, 1}^{2}, Y_{i, 2}^{2}, Y_{i, 3}^{2}, Y_{i, 4}^{2}\right)=0,
\end{aligned}
$$

where $F_{i, j}^{1}, F_{i, j}^{2}$ are given by 


$$
\begin{aligned}
& F_{i, j}^{1}=e^{Y_{i, j}^{1}}-K_{1}+\tilde{V}\left(e^{Y_{i j,}^{1}} ; e^{Y^{1}(\tau)}, e^{Y^{2}(\tau)}\right), \\
& F_{i, j}^{2}=e^{Y_{i, j}^{2}}-K_{2}-\tilde{V}\left(e^{Y_{i, j}^{2}} ; e^{Y^{1}(\tau)}, e^{Y^{2}(\tau)}\right) .
\end{aligned}
$$

In (13) and (14), $\mathrm{Y}_{\mathrm{i}, 1}^{1}, \mathrm{Y}_{\mathrm{i}, 2}^{1}, \mathrm{Y}_{\mathrm{i}, 3}^{1}, \mathrm{Y}_{\mathrm{i}, 4}^{1} ; \mathrm{Y}_{\mathrm{i}, 1}^{2}, \mathrm{Y}_{\mathrm{i}, 2}^{2}, \mathrm{Y}_{\mathrm{i}, 3}^{2}, \mathrm{Y}_{\mathrm{i}, 4}^{2} \quad \mathrm{i}=0,1, \ldots, \mathrm{L}-1$ are unknowns. Newton iteration methods will be used to solve the system (13) and (14). Newton iteration methods require the calculations of Jacobians:

$$
\begin{gathered}
\frac{\partial \mathrm{F}_{\mathrm{i}, \mathrm{j}}^{1}}{\partial \mathrm{Y}_{\mathrm{i}, \mathrm{j}}^{1}}, \frac{\partial \mathrm{F}_{\mathrm{i}, \mathrm{j}}^{1}}{\partial \mathrm{Y}_{\mathrm{i}, \mathrm{j}}^{2}}, \frac{\partial \mathrm{F}_{\mathrm{i}, \mathrm{j}}^{2}}{\partial \mathrm{Y}_{\mathrm{i}, \mathrm{j}}^{1}}, \frac{\partial \mathrm{F}_{\mathrm{i}, \mathrm{j}}^{2}}{\partial \mathrm{Y}_{\mathrm{i}, \mathrm{j}}^{2}} \\
\frac{\partial \mathrm{F}_{\mathrm{i}, \mathrm{j}}^{1}}{\partial \mathrm{Y}_{\mathrm{i}, \mathrm{k}}^{1}}, \frac{\partial \mathrm{F}_{\mathrm{i}, \mathrm{j}}^{1}}{\partial \mathrm{Y}_{\mathrm{i}, \mathrm{k}}^{2}}, \frac{\partial \mathrm{F}_{\mathrm{i}, \mathrm{j}}^{2}}{\partial \mathrm{Y}_{\mathrm{i}, \mathrm{k}}^{1}}, \frac{\partial \mathrm{F}_{\mathrm{i}, \mathrm{j}}^{2}}{\partial \mathrm{Y}_{\mathrm{i}, \mathrm{k}}^{2}} \quad(\mathrm{k} \neq \mathrm{j}),
\end{gathered}
$$

which are given in the appendix.

\section{Numerical implementations}

In the example, we use collocation methods (13), (14) to find the solution of integral equation (6), (7). The result gives the value of the optimal exercise boundaries. With the computational results, the value of American strangle options can computed by solving the partial differential equation (2). The numerical results will be compared with the binomial tree methods (Deng, Ma and Shan (2011)), Crank-Nicolson methods and Kim's methods (Chiarella and Ziogas (2005)). In this example, we set

$$
\mathrm{r}=0.05, \mathrm{q}=0.1, \sigma=0.2, \mathrm{~K}_{1}=1, \mathrm{~K}_{2}=1.5, \mathrm{~T}-\mathrm{t}=1 \text {. }
$$

Table 1 shows that the collocation methods converge to the exact solutions with five-digit accuracy. In particular, the collocation methods have six-digit accuracy for $\mathrm{S}=0.75,1.25$ and 1.75 . The computation used Matlab 7.1 on PC computer with Pentium(R) Dual-Core CPU T4300, 2.10GHZ processor, 1.99GB memory. It took 53.875 seconds using collocation methods to generate the early exercise boundaries, while Chialella and Ziogas (2005) took 195 seconds to generate the early exercise boundaries. Obviously collocation methods are much faster than Chiarella and Ziogas's approach. Fig 2 and Fig 3 provide drawing of the computed early exercise boundaries using collocation methods. Fig 4 and Fig 5 sketch some Greeks.

\section{Concluding remarks}

In this paper we have developed the high-order collocation method for the valuation of American strangle options and determination of the early exercise boundaries. The optimal exercise boundaries were resolved by solving a system of nonlinear integral equations for the optimal using high-accuracy collocation methods. Numerical example showed that our methods are much more efficient than that in the literature. Integral equation approaches, where the numerical solutions of integral equations are the crucial steps, have been widely used to price American options. The adoption of collocation methods to solving the integral equations accelerates the speed of computations. This approach will be further investigated for pricing more complex American strangle options with stochastic volatility and interest rate in this near future.

\section{References}

Alobaidi, G. and R. Mallier. (2002). Laplace transforms and the American straddle. Journal of Applied Mathematics, 2, 121-129.

Black, F. and M. Scholes. (1973). The pricing of options and corporate liabilities. Journal of Political Economy, 81, 637-659.

Boyarchenko, S. (2006). Two-point boundary problems and perpetual American strangles in jump-diffusion models, Working Paper.

Brunner, H. (2004). Collocation Methods for Volterra Integral and Related Functional Equations. Cambridge University Press, Cambridge. http://dx.doi.org/10.1017/CBO9780511543234

Carr, P., Jarrow R. and Myneni R. (1992). Alternative characterizations of American put option. Mathematical Finance, 2, 87-106. http://dx.doi.org/ 10.1111/j.1467-9965.1992.tb00040.x.

Chiarella, C. and A. Ziogas. (2005). Evaluation of American strangles. Journal of Economic Dynamics and Control, 29, 31-62. 
Deng, D., Ma, J. and Shan, Y. (2011). Studies on American strangles pricing and applications. South Finance (in Chinese), 424, 86-89.

Detemple, J. and Tian, W. (2002). The valuation of American options for a class of diffusion processes. Management Science, 48, 917-937. http://dx.doi.org/ 10.1287/mnsc.48.7.917.2815

Detemple, J. and T. Emmerling. (2009). American chooser options. Journal of Economic Dynamics and Control, 33, 128-153.

Elliott, R.J., Myneni, R., Viswanathan, R. (1990). A theorem of El Karoui-Karatzas applied to the American option. Statistics Centre, University of Alberta, Technical Report 91-19.

Huang, J., Subrahmanyam,M. and Yu. G. (1996). Pricing and hedging American options: A recursive integration method. Review of Financial Studies, 9, 277-330. http://dx.doi.org/10.1093/rfs/9.1.277

Jacka, S.D. (1991). Optimal stopping and the American put. Mathematical Finance, 1, 1-14. http://dx.doi.org/ 10.1111/j.1467-9965.1991.tb00007.x

$\mathrm{Ju}, \mathrm{N}$. (1998). Pricing an American option by approximating its early exercise boundary as a multipiece exponential function. Review of Financial Studies, 11, 627-646. http://dx.doi.org/10.1093/rfs/11.3.62

Kim, I.J. (1990). The analytic valuation of American options. Review of Financial Studies, 3, 547-572. http://dx.doi.org/ $10.1093 / \mathrm{rfs} / 3.4 .547$

Ma, J., Xiang, K. and Jiang Y. (2010). An integral equation method with high-order collocation implementations for pricing American put option. International Journal of Economics and Finance, 2, 102-113.

Ma, J. and Zhou Z.R. (2011). High-accuracy integral equation approach for pricing American options with stochastic volatility. International Journal of Economics and Finance, 3, 193-201.

Table 1. Valuation of American strangles

\begin{tabular}{|c|c|c|c|c|c|c|c|}
\hline S & $\begin{array}{c}\text { Binomial } \\
4000\end{array}$ & $\begin{array}{c}\text { Binomial } \\
8000\end{array}$ & $\begin{array}{c}\mathrm{CN} \\
60,000\end{array}$ & $\begin{array}{c}\mathrm{CN} \\
120,000\end{array}$ & $\begin{array}{c}\text { Kim } \\
400\end{array}$ & $\begin{array}{c}\text { Kim } \\
800\end{array}$ & Collocation \\
\hline 0.75 & 0.275647 & 0.275648 & 0.275648 & 0.275648 & 0.275647 & 0.275647 & 0.275649 \\
\hline 1.00 & 0.100326 & 0.100329 & 0.100322 & 0.100319 & 0.100322 & 0.100322 & 0.100335 \\
\hline 1.25 & 0.038561 & 0.038560 & 0.038560 & 0.038560 & 0.038561 & 0.038561 & 0.038568 \\
\hline 1.50 & 0.092335 & 0.092338 & 0.092316 & 0.092314 & 0.092341 & 0.092340 & 0.092348 \\
\hline 1.75 & 0.255633 & 0.255633 & 0.255619 & 0.255619 & 0.255633 & 0.255633 & 0.255636 \\
\hline
\end{tabular}

The Crank-Nicolson (CN): finite difference scheme involved four time steps per day, and involved 60,000 and 120,000 space-nodes, as indicated in the table. The numerical scheme for solving Kim's integral equations used $n=400$ and 800 , respectively, as indicated in the table. The binomial tree methods (Binomial) used 4000 and 8000 steps. The numerics in Column 2 and 3 were given by Deng, Ma and Shan (2011), Columns 4-7 by Chiarella and Ziogas (2005).

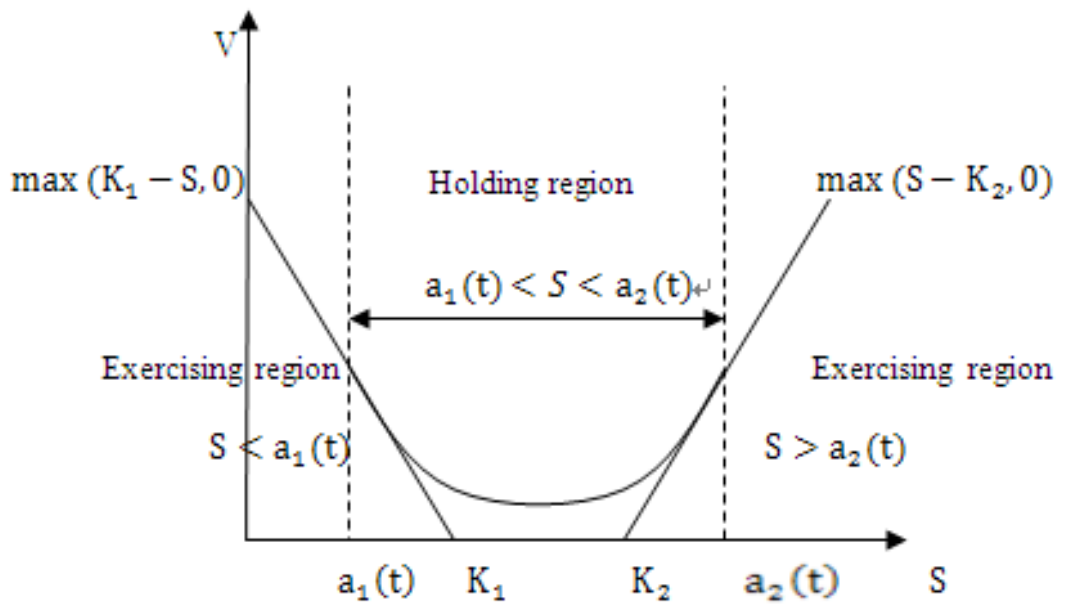

Figure 1. 


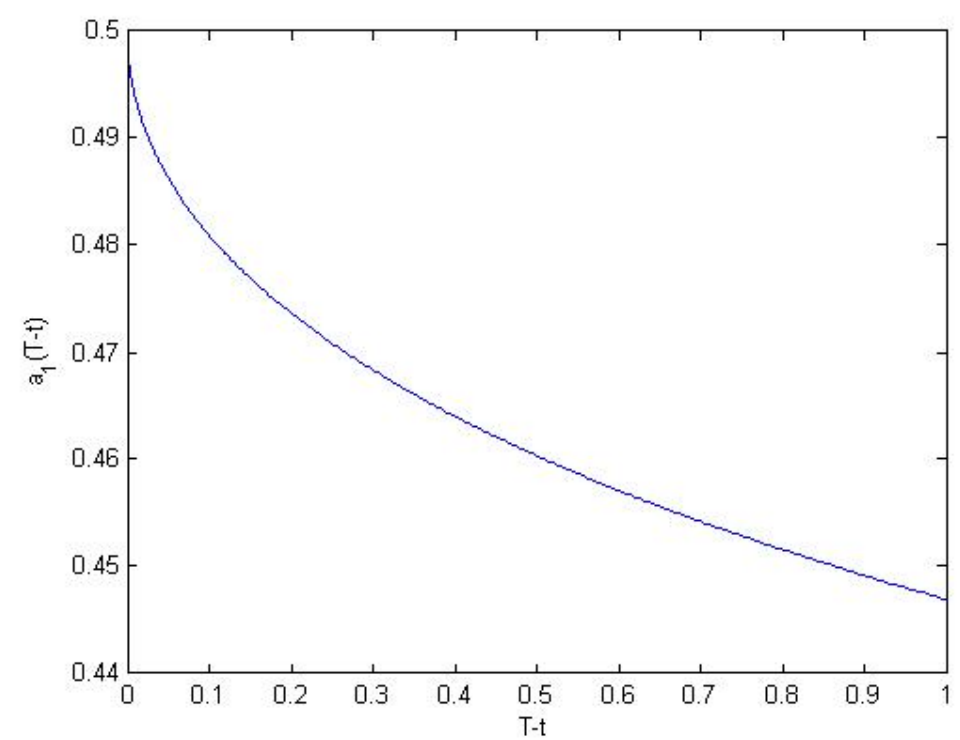

Figure 2. Put-side early exercise boundary for American strangles

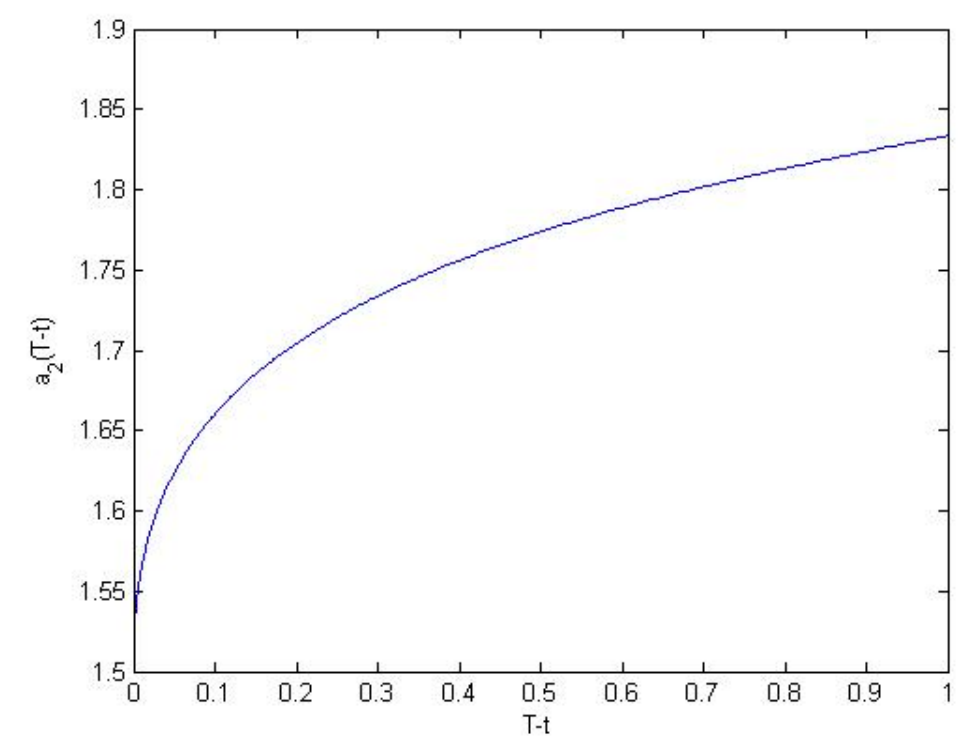

Figure 3. Call-side early exercise boundary for American strangles 


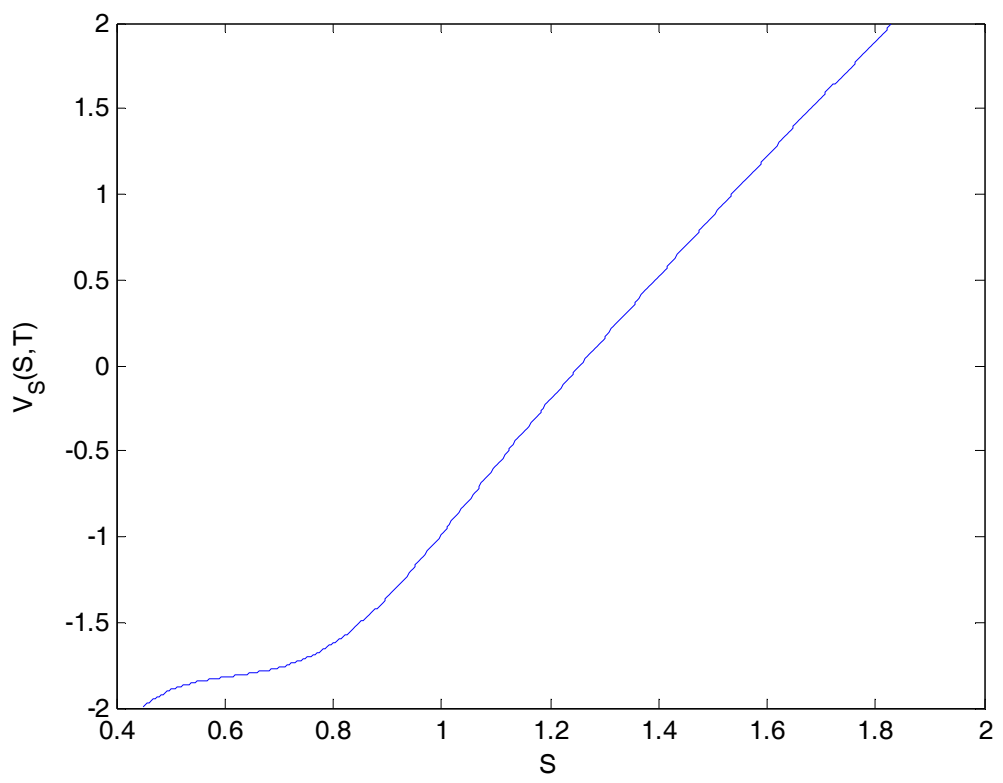

Figure 4. $\Delta$ value for American strangles

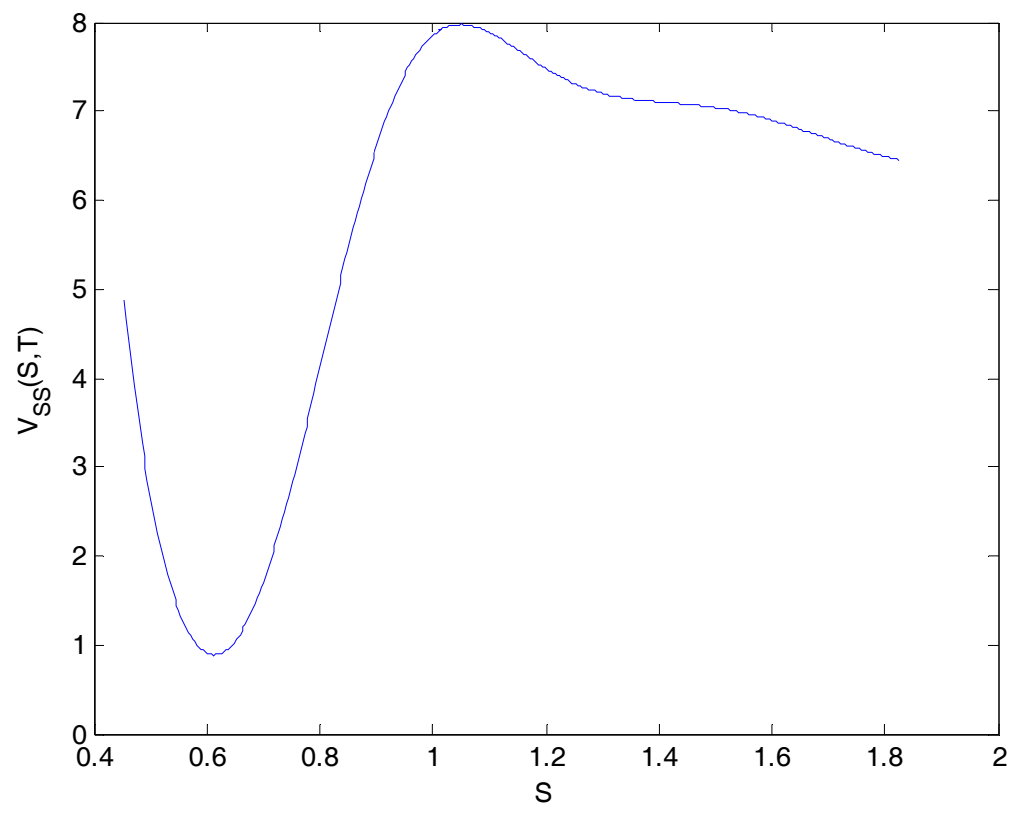

Figure 5. $\Gamma$ value for American strangles 
Appendix 1: The formulas of Jacobians are given by

$$
\begin{aligned}
& \frac{\partial F_{i, j}^{1}}{\partial Y_{i, j}^{1}}=e^{Y_{i, j}^{1}}+e^{Y_{i, j}^{1}-q \tau_{i, j}}\left(N\left(d_{1}\left(e^{Y_{i, j}^{1}}, \tau_{i, j} ; K_{1}\right)\right)+N\left(d_{1}\left(e^{Y_{i, j}^{1}}, \tau_{i, j} ; K_{2}\right)\right)-1\right)
\end{aligned}
$$

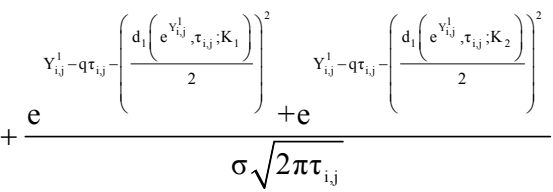

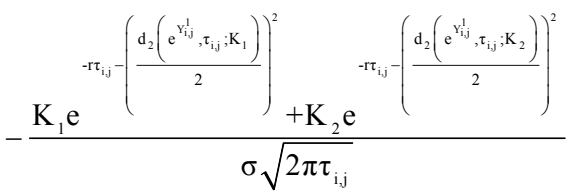

$$
\begin{aligned}
& +\int_{0}^{\tau_{i, j}}\left[\mathrm{qe}^{\mathrm{Y}_{\mathrm{i,j}, \mathrm{i}}^{1}-\mathrm{q}\left(\tau_{\mathrm{i}, \mathrm{j}}-v\right)}\left(\mathrm{Nd}_{1}\left(\left(\mathrm{e}^{\mathrm{Y}_{\mathrm{i}, \mathrm{j}}^{1}}, \tau_{\mathrm{i}, \mathrm{j}}-v ; \delta_{2}(v)\right)\right)+\mathrm{N}\left(\mathrm{d}_{1}\left(\mathrm{e}^{\mathrm{Y}_{\mathrm{i}, \mathrm{j}}^{1}}, \tau_{\mathrm{i}, \mathrm{j}}-v ; \delta_{3}(v)\right)\right)-1\right)\right] \mathrm{d} v
\end{aligned}
$$

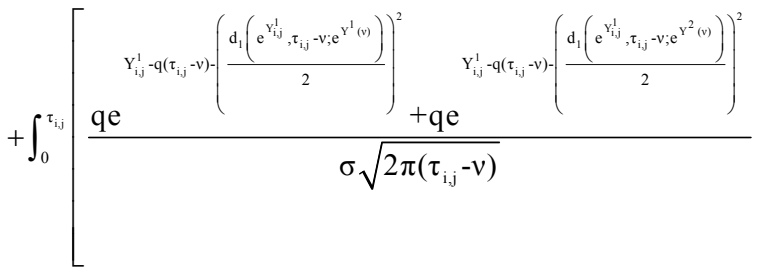

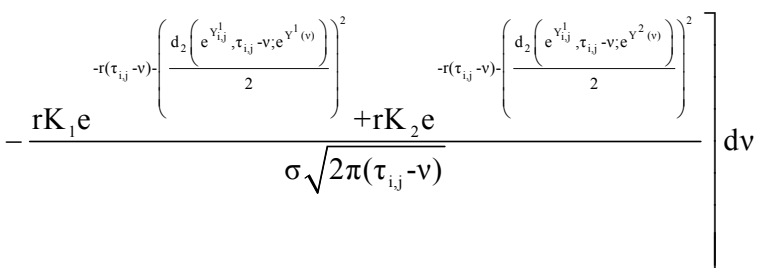

$$
\begin{aligned}
& \frac{\partial \mathrm{F}_{\mathrm{i}, \mathrm{j}}^{2}}{\partial \mathrm{Y}_{\mathrm{i}, \mathrm{j}}^{2}}=\mathrm{e}^{\mathrm{Y}_{\mathrm{ij}}^{2}}+\mathrm{e}^{\mathrm{Y}_{\mathrm{i}, \mathrm{j}}^{2}-\mathrm{q}_{\mathrm{i}, \mathrm{j}}}\left(1-\mathrm{N}\left(\mathrm{d}_{1}\left(\mathrm{e}^{\mathrm{Y}_{\mathrm{ij \textrm {j }}}^{2}}, \tau_{\mathrm{i}, \mathrm{j}}, \mathrm{K}_{1}\right)\right)-\mathrm{N}\left(\mathrm{d}_{1}\left(\mathrm{e}^{\mathrm{Y}_{\mathrm{ij}}^{2}}, \tau_{\mathrm{i}, \mathrm{j}}, \mathrm{K}_{2}\right)\right)\right) \\
& +\int_{0}^{\tau_{i, j}}\left[\mathrm{qe}^{\mathrm{Y}_{\mathrm{ijj}}^{2}-q\left(\tau_{\mathrm{i}, j}-v\right)}\left(1-\mathrm{N}\left(\mathrm{d}_{1}\left(\mathrm{e}^{\mathrm{Y}_{\mathrm{ij}}^{2}}, \tau_{\mathrm{i}, \mathrm{j}}-v, \mathrm{e}^{\mathrm{Y}^{1}(v)}\right)\right)-\mathrm{N}\left(\mathrm{d}_{1}\left(\mathrm{e}^{\mathrm{Y}_{\mathrm{ij}}^{2}}, \tau_{\mathrm{i}, \mathrm{j}}-v, \mathrm{e}^{\mathrm{Y}^{2}(v)}\right)\right)\right)\right] \mathrm{d} v
\end{aligned}
$$

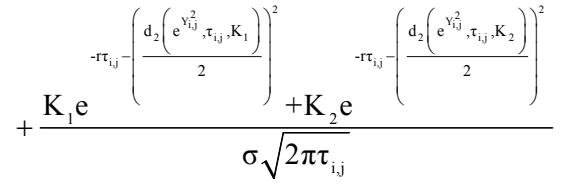

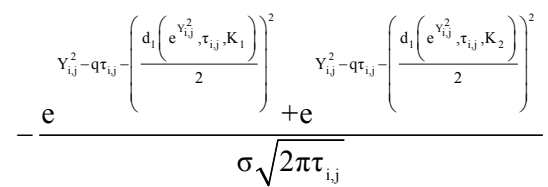

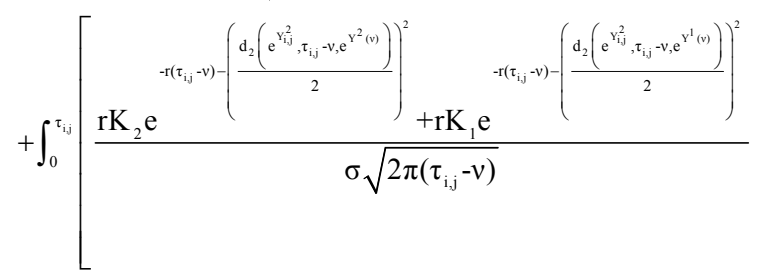

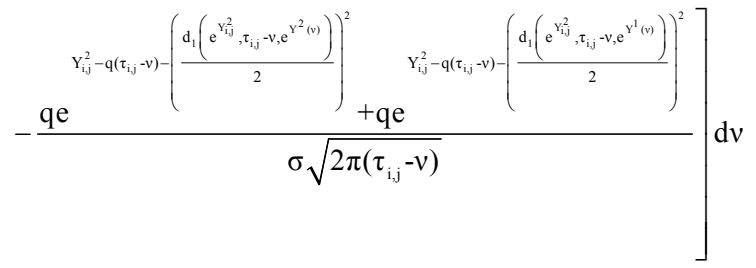


$\frac{\partial \mathrm{F}_{\mathrm{i}, \mathrm{j}}^{1}}{\partial \mathrm{Y}_{\mathrm{i}, \mathrm{j}}^{2}}=0, \frac{\partial \mathrm{F}_{\mathrm{i}, \mathrm{j}}^{2}}{\partial \mathrm{Y}_{\mathrm{i}, \mathrm{j}}^{1}}=0, \frac{\partial \mathrm{F}_{\mathrm{i}, \mathrm{j}}^{1}}{\partial \mathrm{Y}_{\mathrm{i}, \mathrm{k}}^{1}}=0, \frac{\partial \mathrm{F}_{\mathrm{i}, \mathrm{j}}^{1}}{\partial \mathrm{Y}_{\mathrm{i}, \mathrm{k}}^{2}}=0, \frac{\partial \mathrm{F}_{\mathrm{i}, \mathrm{j}}^{2}}{\partial \mathrm{Y}_{\mathrm{i}, \mathrm{k}}^{1}}=0, \frac{\partial \mathrm{F}_{\mathrm{i}, \mathrm{j}}^{2}}{\partial \mathrm{Y}_{\mathrm{i}, \mathrm{k}}^{2}}=0$.

Proof: (The quoted equation numbers are independent of those for the main sections.)

Step 1: Calculate the following partial derivatives:

$$
\begin{aligned}
& \frac{\partial \tilde{\mathrm{V}}\left(\delta_{1}(\tau), \delta_{2}(\tau), \delta_{3}(\tau)\right)}{\partial \delta_{1}(\tau)}=\frac{\partial \tilde{p}_{\tau}\left(\delta_{1}(\tau)\right)}{\partial \delta_{1}(\tau)}+\frac{\partial \int_{0}^{\tau} \tilde{\Phi}_{\tau}^{1}\left(\delta_{2}(v), \delta_{1}(\tau), v\right) \mathrm{d} v}{\partial \delta_{1}(\tau)} \\
& +\frac{\partial \tilde{\mathbf{c}}_{\tau}\left(\delta_{1}(\tau)\right)}{\partial \delta_{1}(\tau)}+\frac{\partial \int_{0}^{\tau} \tilde{\Phi}_{\tau}^{2}\left(\delta_{3}(v), \delta_{1}(\tau), v\right) \mathrm{d} v}{\partial \delta_{1}(\tau)}, \\
& \frac{\partial \tilde{\mathrm{p}}_{\tau}\left(\delta_{1}(\tau)\right)}{\partial \delta_{1}(\tau)}=-\frac{\mathrm{K}_{1} \mathrm{e}^{-\mathrm{r \tau}-\left(\frac{\mathrm{d}_{2}\left(\delta_{1}(\tau), \tau ; \mathrm{K}_{1}\right)}{2}\right)^{2}}}{\delta_{1}(\tau) \sigma \sqrt{2 \pi \tau}}-\mathrm{e}^{-\mathrm{q} \tau} \\
& +\mathrm{e}^{-\mathrm{q} \tau} \mathrm{N}\left(\mathrm{d}_{1}\left(\delta_{1}(\tau), \tau ; \mathrm{K}_{1}\right)\right)+\frac{\mathrm{e}^{-\mathrm{q} \tau-\left(\frac{\mathrm{d}_{1}\left(\delta_{1}(\tau), \tau ; \mathrm{K}_{1}\right)}{2}\right)^{2}}}{\sigma \sqrt{2 \pi \tau}} \\
& \frac{\partial \tilde{\mathbf{c}}_{\tau}\left(\delta_{1}(\tau)\right)}{\partial \delta_{1}(\tau)}=\mathrm{e}^{-q \tau} \mathrm{N}\left(\mathrm{d}_{1}\left(\delta_{1}(\tau), \tau ; \mathrm{K}_{2}\right)\right)+\frac{\mathrm{e}^{-q \tau-\left(\frac{\mathrm{d}_{1}\left(\delta_{1}(\tau), \tau ; \mathrm{K}_{2}\right)}{2}\right)^{2}}}{\sigma \sqrt{2 \pi \tau}}-\frac{\mathrm{K}_{2} \mathrm{e}^{-\tau \tau-\left(\frac{\mathrm{d}_{2}\left(\delta_{1}(\tau), \tau ; \mathrm{K}_{2}\right)}{2}\right)^{2}}}{\delta_{1}(\tau) \sigma \sqrt{2 \pi \tau}} \\
& \frac{\partial \int_{0}^{\tau} \tilde{\Phi}_{\tau}^{1}\left(\delta_{2}(v), \delta_{1}(\tau), v\right) \mathrm{d} v}{\partial \delta_{1}(\tau)}=\int_{0}^{\tau}\left[-\frac{\mathrm{rK}_{1} \mathrm{e}^{-\mathrm{r}(\tau-v)-\left(\frac{\mathrm{d}_{2}\left(\delta_{1}(\tau), \tau-\gamma ; \delta_{2}(v)\right)}{2}\right)^{2}}}{\delta_{1}(\tau) \sigma \sqrt{2 \pi(\tau-v)}}-\mathrm{qe}^{-\mathrm{q}(\tau-v)}\right. \\
& \left.+\mathrm{qe}^{-\mathrm{q}(\tau-v)} \mathrm{N}\left(\mathrm{d}_{1}\left(\delta_{1}(\tau), \tau-v ; \delta_{2}(v)\right)\right)+\frac{\mathrm{qe}^{-\mathrm{q}(\tau-v)-\left(\frac{\mathrm{d}_{1}\left(\delta_{1}(\tau), \tau-\gamma ; \delta_{2}(v)\right)}{2}\right)^{2}}}{\sigma \sqrt{2 \pi(\tau-v)}}\right] \mathrm{d} v \\
& \frac{\partial \int_{0}^{\tau} \tilde{\Phi}_{\tau}^{2}\left(\delta_{3}(v), \delta_{1}(\tau), v\right) \mathrm{d} v}{\partial \delta_{1}(\tau)}=\int_{0}^{\tau}\left[\mathrm{qe} \mathrm{e}^{-\mathrm{q}(\tau-v)} \mathrm{N}\left(\mathrm{d}_{1}\left(\delta_{1}(\tau), \tau-v ; \delta_{3}(v)\right)\right)\right. \\
& \left.+\frac{\mathrm{qe}^{-\mathrm{q}(\tau-v)-\left(\frac{\mathrm{d}_{1}\left(\delta_{1}(\tau), \tau-\tau ; \delta_{3}(v)\right)}{2}\right)^{2}}}{\sigma \sqrt{2 \pi(\tau-v)}}-\frac{\mathrm{rK}_{2} \mathrm{e}^{-\mathrm{r}(\tau-v)-\left(\frac{\mathrm{d}_{2}\left(\delta_{1}(\tau), \tau-\tau, \delta_{3}(v)\right)}{2}\right)^{2}}}{\delta_{1}(\tau) \sigma \sqrt{2 \pi(\tau-v)}}\right] \mathrm{d} v \\
& \frac{\partial \tilde{\mathrm{V}}\left(\delta_{1}(\tau), \delta_{2}(\tau), \delta_{3}(\tau)\right)}{\partial \delta_{1}(\tau)}=\mathrm{e}^{-\mathrm{q} \tau}\left(\mathrm{N}\left(\mathrm{d}_{1}\left(\delta_{1}(\tau), \tau ; \mathrm{K}_{1}\right)\right)+\mathrm{N}\left(\mathrm{d}_{1}\left(\delta_{1}(\tau), \tau ; \mathrm{K}_{2}\right)\right)-1\right) \\
& +\frac{\mathrm{e}^{-q \tau\left(\frac{\mathrm{d}_{1}\left(\delta_{1}(\tau), \tau ; K_{1}\right)}{2}\right)^{2}}+\mathrm{e}^{-q \tau-\left(\frac{\mathrm{d}_{1}\left(\delta_{1}(\tau), \tau, \mathrm{K}_{2}\right)}{2}\right)^{2}}}{\sigma \sqrt{2 \pi \tau}} \\
& -\frac{\mathrm{K}_{1} \mathrm{e}^{-\mathrm{r \tau}-\left(\frac{\mathrm{d}_{2}\left(\delta_{1}(\tau) \tau, \tau, K_{1}\right)}{2}\right)^{2}}+\mathrm{K}_{2} \mathrm{e}^{-\tau-\left(\frac{\mathrm{d}_{2}\left(\delta_{1}(\tau), \tau K_{2}\right.}{2}\right)^{2}}}{\delta_{1}(\tau) \sigma \sqrt{2 \pi \tau}} \\
& +\int_{0}^{\tau}\left[\mathrm{qe}^{-q(\tau-v)}\left(\mathrm{N}\left(\mathrm{d}_{1}\left(\delta_{1}(\tau), \tau-v ; \delta_{2}(v)\right)\right)+\mathrm{N}\left(\mathrm{d}_{1}\left(\delta_{1}(\tau), \tau-v ; \delta_{3}(v)\right)\right)-1\right)\right] \mathrm{d} v
\end{aligned}
$$




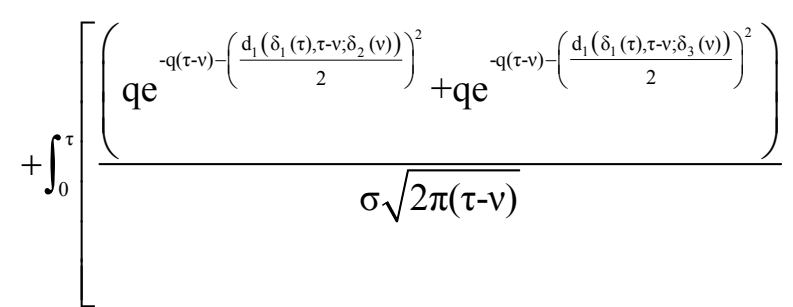

$$
\left.-\frac{\mathrm{rK}_{1} \mathrm{e}^{-\mathrm{r}(\tau-v)-\left(\frac{\mathrm{d}_{2}\left(\delta_{1}(\tau), \tau-v ; \delta_{2}(v)\right)}{2}\right)^{2}}+\mathrm{rK}_{2} \mathrm{e}^{-\mathrm{r}(\tau-v)-\left(\frac{\mathrm{d}_{2}\left(\delta_{1}(\tau), \tau-v ; \delta_{3}(v)\right)}{2}\right)^{2}}}{\delta_{1}(\tau) \sigma \sqrt{2 \pi(\tau-v)}}\right] \mathrm{d} v
$$

Step 2: We study the case $\mathrm{k}=\mathrm{j}$.

$$
\frac{\partial F_{i, j}^{1}}{\partial Y_{i, j}^{1}}=e^{Y_{i, j}^{1}}+\frac{\partial \tilde{V}\left(e^{Y_{i, j}^{1}} ; e^{Y^{1}(\tau)}, e^{Y^{2}(\tau)}\right)}{\partial Y_{i, j}^{1}} .
$$

We need to calculate the second term on the right-hand side of (7):

$$
\begin{aligned}
\frac{\partial \tilde{\mathrm{V}}\left(\mathrm{e}^{\mathrm{Y}_{\mathrm{i}, j}^{1}} ; \mathrm{e}^{\mathrm{Y}^{1}(\tau)}, \mathrm{e}^{\mathrm{Y}^{2}(\tau)}\right)}{\partial \mathrm{Y}_{\mathrm{i}, \mathrm{j}}^{1}}= & \left.\frac{\partial \tilde{\mathrm{V}}\left(\delta_{1}\left(\tau_{\mathrm{i}, \mathrm{j}}\right) ; \mathrm{e}^{\mathrm{Y}^{1}(\tau)}, \mathrm{e}^{\mathrm{Y}^{2}(\tau)}\right)}{\partial \delta_{1}\left(\tau_{\mathrm{i}, \mathrm{j}}\right)}\right|_{\delta_{1}\left(\tau_{\mathrm{i}, \mathrm{j}}\right)=\mathrm{e}^{\mathrm{Y}_{\mathrm{i}, \mathrm{j}}^{1}}} \cdot \frac{\mathrm{de}^{\mathrm{Y}_{\mathrm{i}, \mathrm{j}}^{1}}}{\mathrm{d \textrm {Y } _ { \mathrm { i } , \mathrm { j } } ^ { 1 }}} \\
& +\int_{0}^{\tau_{\mathrm{i}, \mathrm{j}}}\left[\frac{\partial \tilde{\Phi}_{\tau_{\mathrm{i}, \mathrm{j}}}^{1}\left(\mathrm{e}^{\mathrm{Y}^{1}(v)}, \mathrm{e}^{\mathrm{Y}_{\mathrm{i}, \mathrm{j}}^{1}}, v\right)}{\partial \mathrm{e}^{\mathrm{Y}^{1}(v)}} \cdot \frac{\partial \mathrm{e}^{\mathrm{Y}^{1}(v)}}{\partial \mathrm{Y}_{\mathrm{i}, \mathrm{j}}^{1}}\right] \mathrm{d} v
\end{aligned}
$$

The first term on the right-hand side of (8) can be given directly by formula (6). Now we calculate the second term. Since

$$
\mathrm{Y}^{1}(v)=\sum_{\mathrm{k}=1}^{4} \mathrm{Y}_{\mathrm{i}, \mathrm{k}}^{1} 1_{\mathrm{i}, \mathrm{k}}(v), v \in\left[\tau_{\mathrm{i}}, \tau_{\mathrm{i}+1}\right], \text { when } v \in\left[0, \tau_{i}\right), \frac{\partial \mathrm{Y}^{1}(v)}{\partial \mathrm{Y}_{\mathrm{i}, \mathrm{j}}^{1}}=0,
$$

we have

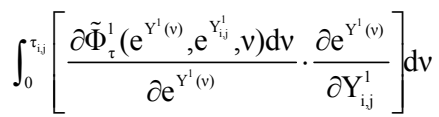

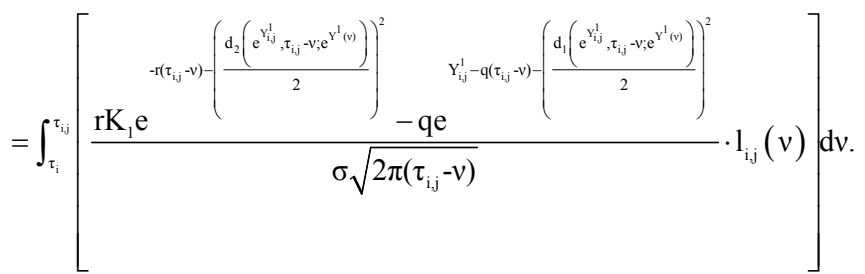

We can see that $(9) \approx O(\Delta \tau)$, where $\Delta \tau=\tau_{i+1}-\tau_{i}$. Similarly to the calculation of $(9)$, we can obtain

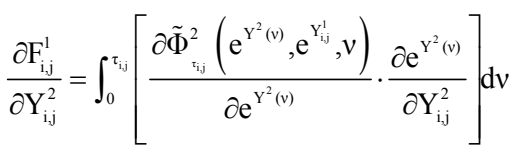

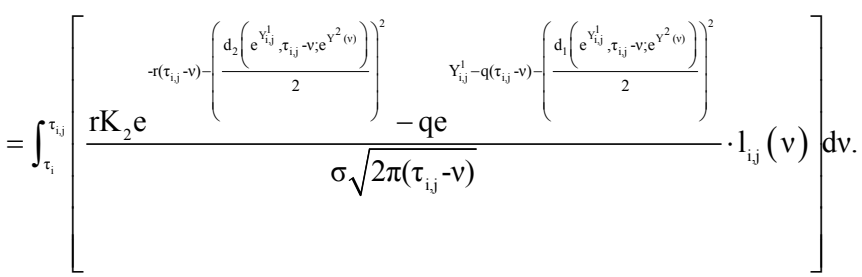

Obviously, $(10) \approx \mathrm{O}(\Delta \tau)$, where $\Delta \tau=\tau_{i+1}-\tau_{i}$. Now we calculate

$$
\frac{\partial \mathrm{F}_{\mathrm{i}, \mathrm{j}}^{2}}{\partial \mathrm{Y}_{\mathrm{i}, \mathrm{j}}^{2}}=\mathrm{e}^{\mathrm{Y}_{\mathrm{i}, \mathrm{j}}^{2}}-\frac{\partial \tilde{\mathrm{V}}\left(\mathrm{e}^{\mathrm{Y}_{\mathrm{ij}}^{2}} ; \mathrm{e}^{\mathrm{Y}^{1}(\tau)}, \mathrm{e}^{\mathrm{Y}^{2}(\tau)}\right)}{\partial \mathrm{Y}_{\mathrm{i}, \mathrm{j}}^{2}} .
$$


with

$$
\begin{aligned}
& \frac{\partial \tilde{V}\left(e^{Y_{i, j}^{2}} ; e^{Y^{1}(\tau)}, e^{Y^{2}(\tau)}\right)}{\partial Y_{i, j}^{2}}=\left.\frac{\partial \tilde{V}\left(\delta_{1}\left(\tau_{i, j}\right) ; e^{Y^{1}(\tau)}, e^{Y^{2}(\tau)}\right)}{\partial \delta_{1}\left(\tau_{i, j}\right)}\right|_{\delta_{1}\left(\tau_{i, j}\right)=e^{Y_{i, j}^{2}}} \cdot \frac{\mathrm{de}^{Y_{i, j}^{2}}}{d Y_{i, j}^{2}}
\end{aligned}
$$

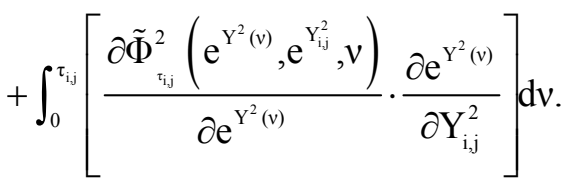

In the same manner, the first term in the right-hand side of (12) can be calculated by formula (6). Using the same method for calculation of (9), we can get

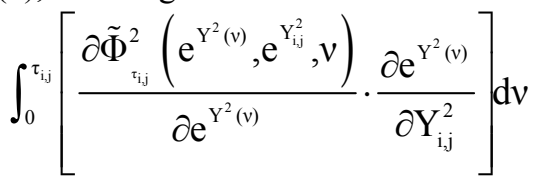

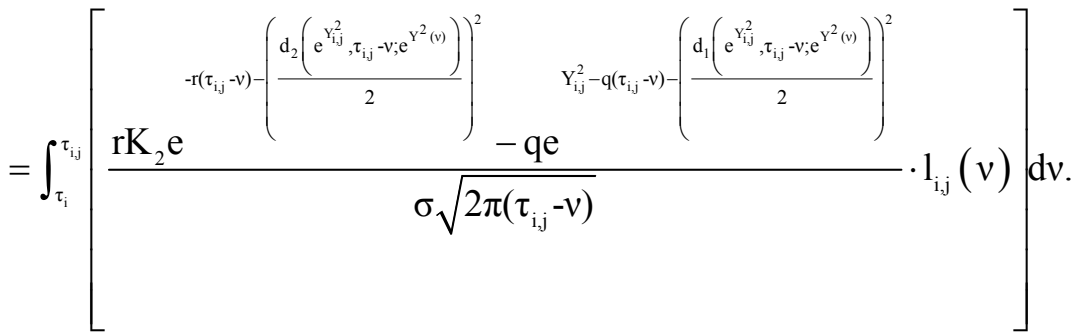

$\operatorname{Again}(13) \approx \mathrm{O}(\Delta \tau)$, where $\Delta \tau=\tau_{i+1}-\tau_{i}$.

Using the same methods, we can obtain that

$$
\begin{aligned}
& \frac{\partial \mathrm{F}_{\mathrm{i}, \mathrm{j}}^{2}}{\partial \mathrm{Y}_{\mathrm{i}, \mathrm{j}}^{1}}=\int_{0}^{\tau_{\mathrm{i}, j}}\left[\frac{\partial \tilde{\Phi}_{\mathrm{\tau}_{\mathrm{i}, j}}^{1}\left(\mathrm{e}^{\mathrm{Y}^{1}(v)}, \mathrm{e}^{\mathrm{Y}_{\mathrm{ij}}^{2}}, v\right)}{\partial \mathrm{e}^{\mathrm{Y}^{1}(v)}} \cdot \frac{\partial \mathrm{e}^{\mathrm{Y}^{1}(v)}}{\partial \mathrm{Y}_{\mathrm{i}, \mathrm{j}}^{1}}\right] \mathrm{d} v
\end{aligned}
$$

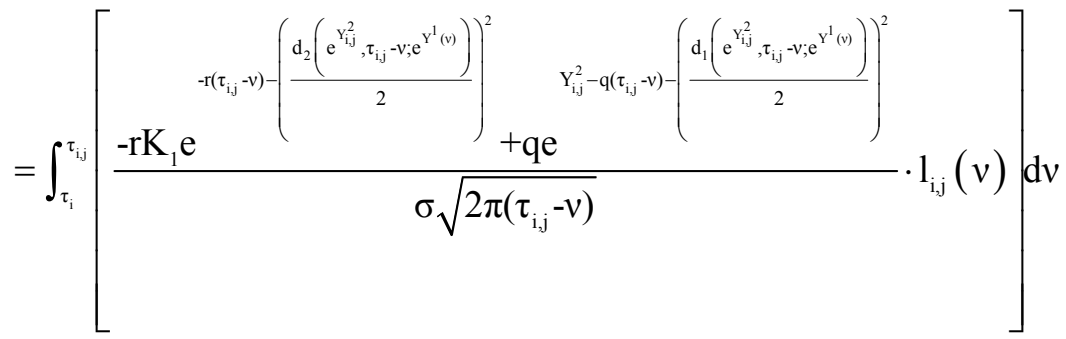

which is proportional to $\mathrm{O}(\Delta \tau)$, where $\Delta \tau=\tau_{i+1}-\tau_{i}$.

Step 3: We study the case $\mathrm{k} \neq \mathrm{j}$.

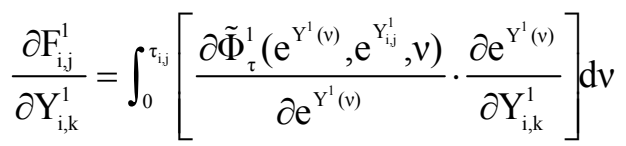

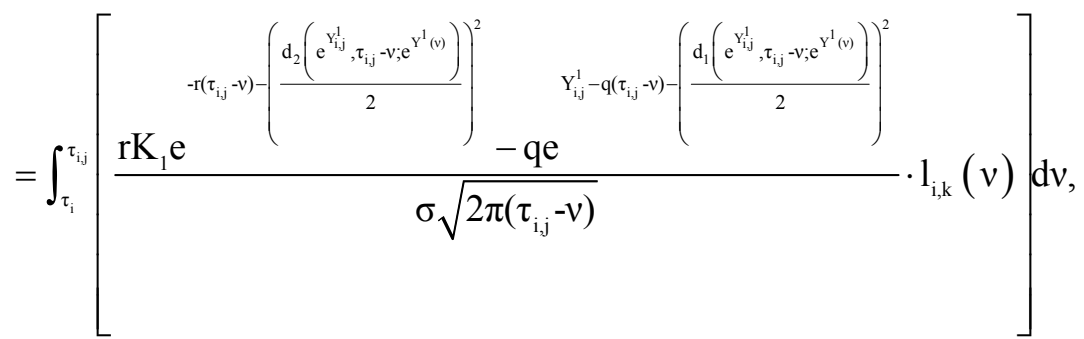




$$
\begin{aligned}
& \frac{\partial \mathrm{F}_{\mathrm{i}, \mathrm{j}}^{1}}{\partial \mathrm{Y}_{\mathrm{i}, \mathrm{k}}^{2}}=\int_{0}^{\tau_{\mathrm{i}, \mathrm{j}}}\left[\frac{\partial \tilde{\Phi}_{\tau}^{2}\left(\mathrm{e}^{\mathrm{Y}^{2}(v)}, \mathrm{e}^{\mathrm{Y}_{\mathrm{ij}}^{1}}, v\right)}{\partial \mathrm{e}^{\mathrm{Y}^{2}(v)}} \cdot \frac{\partial \mathrm{e}^{\mathrm{Y}^{2}(v)}}{\partial \mathrm{Y}_{\mathrm{i}, \mathrm{k}}^{2}}\right] \mathrm{d} v
\end{aligned}
$$

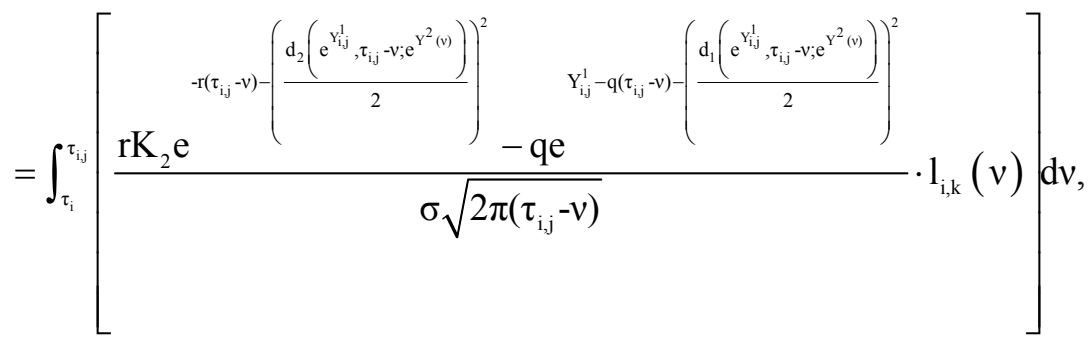

$$
\begin{aligned}
& \frac{\partial \mathrm{F}_{\mathrm{i}, \mathrm{j}}^{2}}{\partial \mathrm{Y}_{\mathrm{i}, \mathrm{k}}^{1}}=\int_{0}^{\tau_{\mathrm{i}, \mathrm{j}}}\left[\frac{\partial \tilde{\Phi}_{\tau}^{1}\left(\mathrm{e}^{\mathrm{Y}^{1}(v)}, \mathrm{e}^{\mathrm{Y}_{\mathrm{i}, \mathrm{j}}^{2}}, v\right)}{\partial \mathrm{e}^{\mathrm{Y}^{1}(v)}} \cdot \frac{\partial \mathrm{e}^{\mathrm{Y}^{1}(v)}}{\partial \mathrm{Y}_{\mathrm{i}, \mathrm{k}}^{1}}\right] \mathrm{d} v
\end{aligned}
$$

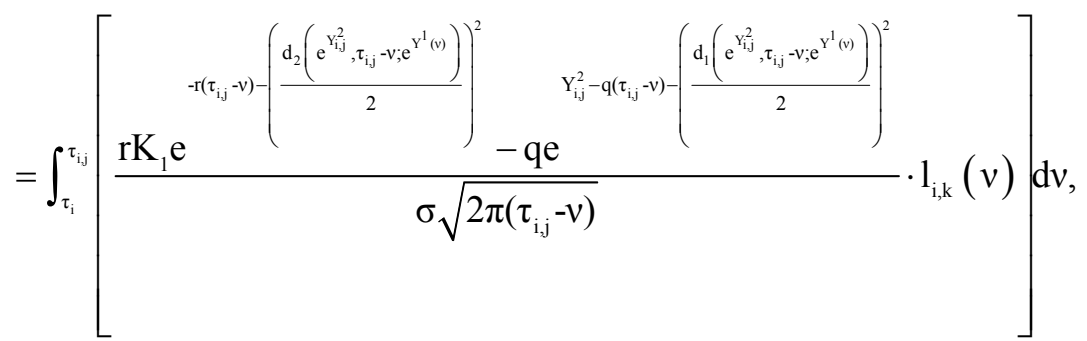

$$
\begin{aligned}
& \frac{\partial \mathrm{F}_{\mathrm{i}, \mathrm{j}}^{2}}{\partial \mathrm{Y}_{\mathrm{i}, \mathrm{k}}^{2}}=\int_{0}^{\tau_{\mathrm{i}, \mathrm{j}}}\left[\frac{\partial \tilde{\Phi}_{\tau}^{2}\left(\mathrm{e}^{\mathrm{Y}^{2}(v)}, \mathrm{e}^{\mathrm{Y}_{\mathrm{i}, \mathrm{j}}^{2}}, v\right)}{\partial \mathrm{e}^{\mathrm{Y}^{2}(v)}} \cdot \frac{\partial \mathrm{e}^{\mathrm{Y}^{2}(v)}}{\partial \mathrm{Y}_{\mathrm{i}, \mathrm{k}}^{2}}\right] \mathrm{d} v
\end{aligned}
$$

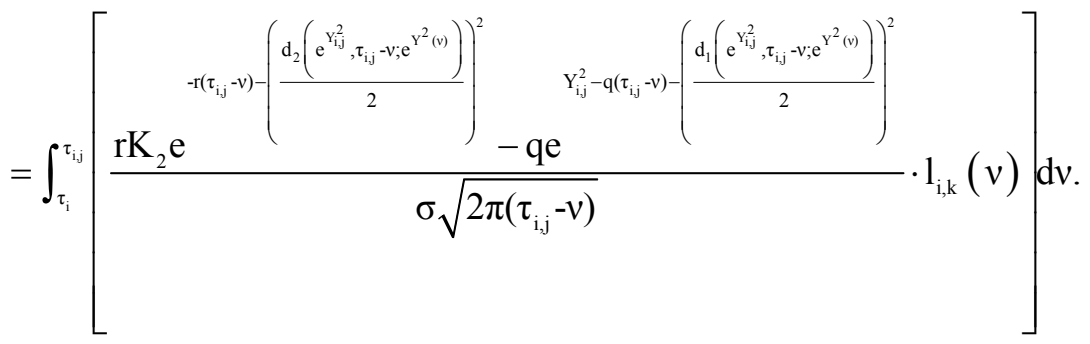

From the previous computational results, we can see that (15)---(18) are all proportional to $\mathrm{O}(\Delta \tau)$, where $\Delta \tau=\tau_{i+1}-\tau_{i}$. 